\title{
Heart Rate Variability as an Indicator of Autonomic Nervous System Disturbance in Behcet's Disease
}

\author{
Imtinene Ben Mrad (D) \\ Melek Ben Mrad (D) ${ }^{2}$ \\ Bouthaina Besbes' \\ Ihsen Zairi (D) \\ Nofeil Ben Kahla (D) \\ Sofien Kamoun (D) ${ }^{\prime}$ \\ Khadija Mzoughi (D' \\ Sondos Kraiem'
}

'Cardiology Department, Habib Thameur Hospital, Tunis, Tunisia; ${ }^{2}$ Cardiovascular Surgery Department, Rabta Hospital, Tunis, Tunisia

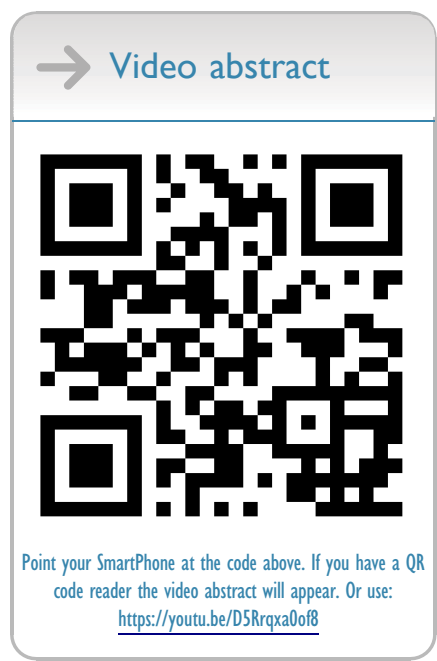

Correspondence: Imtinene Ben Mrad Cardiology Department, Habib Thameur Hospital, App 2b, Bloc 12, cite Olympique, Tunis, 1003, Tunisia Tel +21620156852

Email imtinenebenmradI2@gmail.com
Background: Behçet's disease (BD) is a multisystemic vasculiti where cardiac involvement is not common. Previous studies have shown that the incidence of ventricular arrhythmia and sudden cardiac death (SCD) is higher in patients with BD than in the healthy population. Among various possible explanations, autonomic nervous system (ANS) dysfunction has been suggested. Few studies have evaluated ANS function in patients with BD, and the results obtained are controversial.

Objective: We aimed to assess cardiac autonomic function by heart rate variability (HRV) in patients with $\mathrm{BD}$; to evaluate circadian changes in HRV; and to study the relationship between HRV parameters and disease parameters.

Methods: Thirty-six patients with Behçet's disease (median age 42 years, 28 men) and 36 age- and sex-matched healthy volunteers were included. HRV analyses were performed in the time and frequency domains for the entire 24-hour period and for the daytime and nighttime periods. BD activity was studied with Behcet's disease current activity form (BDCAF).

Results: Patients with BD had significantly lower values of SDNN compared to controls. PNN50, RMSSD, and HF components mean values were significantly reduced in patients than in controls implying parasympathetic impairment. LF was comparable between the two groups, whereas LF/HF ratio was significantly higher in BD group. The circadian rhythm of HRV was preserved in patient group. There was no significant correlation of CRP or disease duration with HRV indices in Behçet's subjects. BDCAF score was found to be negatively correlated with LF/ HF.

Conclusion: Patients with BD, despite no cardiovascular involvement, have reduced parasympathetic activity compared with controls. However, circadian rhythms of autonomic function were preserved. As known, there is strong evidence for the role of the ANS in the pathogenesis of ventricular arrhythmias. Thus, being a practical tool, HRV can be an interesting approach for the rhythmic follow-up of BD patients.

Keywords: Behcet's disease, autonomic nervous system, heart rate variability, disease activity

\section{Introduction}

Behcet's disease (BD) is a chronic, relapsing, multisystemic vasculitis of unknown aetiology. ${ }^{1}$ The disease usually starts around the third-fourth decade of life. ${ }^{2} \mathrm{BD}$ is more frequent in populations along the ancient silk route extending from East Asia to countries in the Middle East and the Mediterranean. ${ }^{3}$

Previous studies have shown that ventricular arrhythmia incidence and sudden cardiac death $(\mathrm{SCD})$ are higher in patients with $\mathrm{BD}$ than in the healthy population. ${ }^{4,5}$ It is well established that an impairment of the cardiac autonomic nervous system (ANS) under certain circumstances can precipitate or trigger life- 
threatening ventricular arrhythmias. ${ }^{6}$ Although the exact cause of increased SCD in patients with BD is unknown, cardiac autonomic dysfunction may be one of the potential explanations.

The assessment of heart rate variability (HRV), which is the variability of normal sinus beat intervals, is widely used as a noninvasive method to assess the functioning of the autonomic nervous system and is a reliable method for detecting altered autonomic control of the heart and predicting any adverse outcomes, such as arrhythmias and sudden cardiac death. ${ }^{7}$ The impairment of the autonomic nervous system in patients with BD is still a matter of debate, although multiple approaches (including timedomain, frequency-domain heart rate variability, and heart rate turbulence variability analyses) have been used to ascertain ANS impairment. ${ }^{8}$

In the literature, there is scarcity of studies that have assessed ANS function in BD patients, with conflicting results and different degrees of involvement. ${ }^{8}$ The circadian profile of heart variability has not been investigated in patients with BD.

Due to the controversial results of these studies and the absence of studies on HRV in BD patients in Tunisia, we aimed to assess cardiac autonomic function by heart rate variability in patients with $\mathrm{BD}$ and matched controls; to evaluate circadian changes in HRV; and to investigate the relationship of autonomic function parameters with activity disease score, disease duration and inflammatory parameters.

\section{Research Design and Methods Study Design}

We conducted a prospective cross-sectional comparative pilot study.

\section{Study Population}

Patients with Behcet's disease were consecutively recruited from the Internal Medicine Department of Habib Thameur Hospital between January 2020 and December 2020. Healthy volunteer subjects were recruited from the staff hospital and matched 1:1 for sex and age to BD patients.

\section{Inclusion Criteria for the Behcet's Disease Group}

BD was diagnosed according to the International Study Group for Behcet's Disease (ISGBD) criteria. ${ }^{9}$

\section{Inclusion Criteria for the Control Group}

The control group included healthy volunteers who had no history of cardiac disease, no other organ-related disease, normal physical examination and electrocardiogram results. None of the healthy volunteers used any medications.

\section{Exclusion Criteria}

Patients with hypertension, diabetes mellitus, chronic kidney or chronic liver disease, coronary artery disease, pulmonary artery hypertension, arrhythmia, systolic heart failure, moderate or severe valve disease, any chronic or inflammatory disease, thyroid dysfunction, $\mathrm{QRS} \geq 120 \mathrm{~ms}$ or bundle branch block, or an intake of drugs interfering with ANS function were excluded. Participants with holter recordings lasting less than 18 hours, participants with less than $80 \%$ of the recording suitable for analysis and those with holter recordings demonstrating a nonsinus rhythm, sick sinus syndrome, atrioventricular block, or nonsinus beats comprising more than $20 \%$ of the total number of beats were also excluded from the study.

\section{Clinical Evaluation}

All participants underwent history-taking for demographic information (age, sex) and measurements of body weight, height, body mass index (BMI) and blood pressure.

In light of our study, we collected all the clinical findings relative to $\mathrm{BD}$ diagnosis at baseline and during the patient follow-up, including disease duration (expressed in months and calculated according to the first hospital admission at which all criteria were met), clinical features such as oral, genital, or perineal aphthosis, cutaneous manifestations, ocular lesions, skin lesions, gastrointestinal involvement, neurological involvement, vascular involvement, arthritis) and prescribed medication.

\section{Disease Activity Assessment}

The Behçet's Disease Current Activity Form (BDCAF) score was used to assess disease activity. ${ }^{10}$ The disease is considered in active phase if the score is of 4 or above.

\section{Heart Rate Variability Analysis}

All participants underwent 24-hour Holter monitoring. Holter recordings were interpreted using ELA MEDICAL SYNE- SCOPE by a specialist cardiologist (SK). The classification of beats was manually checked and corrected if necessary. HRV analyses were performed in both time and frequency domains for the entire 24-hour period and for daytime (06:00-22:00) and night-time 
(22:00-06:00) periods. In the time domain, SDNN (standard deviation of all RR intervals, in milliseconds), PNN50 (percentage of adjacent NN intervals differing by more than $50 \mathrm{~ms}$, in percentage) and RMSSD (root meansquare differences between consecutive RR intervals of the entire recording, in milliseconds) were measured. In the frequency domain, LF (low-frequency power, in $\mathrm{ms}^{2}$ ), HF (high-frequency power, in $\mathrm{ms}^{2}$ ), and LF/HF (the ratio between the powers in the LF and HF bands) were measured.

RMSSD, PNN50 and HF reflect parasympathetic activity, while LF represents sympathetic activity. The LF/HF power ratio estimates the sympathovagal balance, and SDNN reflects global heart rate variability. ${ }^{11}$

\section{Laboratory Investigations}

Fasting blood glucose, total cholesterol, triglyceride, highdensity lipoprotein, haemoglobin, creatinine and serum C-reactive protein (CRP) levels were measured from venous blood drawn from all participants following an 8 $h$ fast.

\section{Statistical Methods}

Data were recorded and analysed using SPSS software version 26. We calculated numbers and frequencies (percentages) for categorical variables.

For quantitative variables, we checked the normality of the distribution by the Shapiro-Wilk test. The test showed that heart rate variability data distribution was nonGaussian and hence they were described as median, 25th and 75th percentile (interquartile range). For normally distributed continuous data, we calculated central tendency (mean) and dispersion (standard deviation (SD)) and total range of the sample (extreme values).

Differences in variables between the two groups were tested using Student's $t$-test for the data with parametric distribution. The comparison of HRV parameters between the $\mathrm{BD}$ group and the control group was made with the non-parametric Mann-Whitney test. The comparison of daytime and nighttime HRV parameters values in the BD group was made with the non-parametric Wilcoxon test. Additionally, for the analysis of the association between disease parameters (activity status, CRP, disease duration) and HRV parameters, the non-parametric Spearman correlation was applied. A two-tailed level of 0.05 was considered statistically significant.

\section{Ethical Considerations}

The study protocol was approved by the Ethics Committee of the Habib Thameur Hospital (approval number HTHEC-2020-117), and it was performed according to the principles of the Declaration of Helsinki. All participants provided written informed consent.

\section{Results \\ Baseline Characteristics}

During the study period, 36 Behcet's disease patients (28 men, 8 women) with a median age of 42 years and 36 healthy volunteer subjects were enrolled. The two groups were matched according to age and sex and were comparable regarding body mass index, systolic and diastolic blood pressure values. There was no difference in biological parameters except for CRP $(\mathrm{p}=0.007)$ as well as and in echocardiographic ejection fraction $(61 \pm 5 \%$ vs $62 \pm 5 \%$; $\mathrm{P}=0.140$ ) between the two groups.

In the Behçet group, the median age at diagnosis was 35 years (20-35), with an estimated mean disease duration of $104.7 \pm 101.8$ months (minimum 8, maximum 336 months). Regarding the clinical profile, most patients manifested oral ulcers $(\mathrm{n}=34,94.4 \%)$, followed by skin lesions $(n=29,80.6 \%)$, genital ulcers $(n=28,77.8 \%)$, and ocular involvement $(\mathrm{n}=26,72.2 \%)$.

The Behcet Disease Current Activity Form (BDCAF) score varied between 0 and 4 with a median of 1.2 . Based on the BDCAF scoring, $88.55 \%$ of patients were in remission and $19.45 \%$ had active Behçet disease (score of 4 and above). Regarding prescribed medication, $80.6 \%$ of the patients were under systemic corticosteroids, all were taking colchicine, and $47.2 \%$ were receiving immunosupressors (76.5\% azathioprine, $23.5 \%$ cyclosporine).

The demographic and clinical characteristics and laboratory findings of the study group are shown in Table 1.

\section{Heart Rate Variability Values}

Time and frequency-domain HRV parameters were analysed for the entire 24-hour period and separately for daytime and nighttime periods. In the 24-hour analysis, the SDNN value was significantly lower in the patient group compared with healthy control group $(\mathrm{p}=0.007)$. Similarly, RMSSD, PNN50 and HF component; indicators of parasympathetic activity; were also significantly lower in the BD group as compared to the control group $(\mathrm{p}=0.001, \mathrm{p}<0.001$ and $\mathrm{p}=0.001$, respectively). Indice of 
Table I Demographics, Clinical and Biological Characteristics of the Studied Groups

\begin{tabular}{|c|c|c|c|}
\hline Variables & Behçet's Group $(n=36)$ & Control Group $(n=36)$ & $P$ value \\
\hline Age (years) ${ }^{\#}$ & $42(32-50)$ & $43(32-50)$ & 0.98 \\
\hline Male/female (n) & $28 / 8$ & $28 / 8$ & \\
\hline BMI $\left(\mathrm{kg} / \mathrm{m}^{2}\right)$ & $23.9 \pm 4.2$ & $22.1 \pm 5.2$ & 0.1 \\
\hline $\mathrm{SBP}(\mathrm{mm} \mathrm{Hg})^{\#}$ & $120(115-122.5)$ & $120(110-120)$ & 0.1 \\
\hline $\mathrm{DBP}(\mathrm{mm} \mathrm{Hg}){ }^{\#}$ & $80(77.5-85)$ & $75(70-80)$ & 0.06 \\
\hline Disease duration (months)* & $104.7 \pm 101.8$ & NA & \\
\hline BDCAF score s $^{\#}$ & 1.2 & NA & \\
\hline Active disease (yes/no) & $7 / 29$ & NA & \\
\hline $\operatorname{CRP}(\mathrm{mg} /)^{*}$ & $7.2 \pm 9.6$ & $2.7 \pm 1.7$ & 0.007 \\
\hline Fasting glucose $(\mathrm{g} / \mathrm{l})^{*}$ & $0.9 \pm 0.2$ & $0.9 \pm 0.1$ & 0.6 \\
\hline Creatinine $(\mathrm{mg} / \mathrm{l})^{*}$ & $7.5 \pm 1.5$ & $7.3 \pm 1.3$ & 0.5 \\
\hline Hemoglobin $(\mathrm{g} / \mathrm{dl})^{*}$ & $13.7 \pm \mid .4$ & $13.8 \pm 1.2$ & 0.8 \\
\hline Total Cholesterol $(\mathrm{mg} / \mathrm{dl})^{*}$ & $173.3 \pm 35.0$ & $161.2 \pm 37$ & 0.1 \\
\hline Triglyceride $(\mathrm{mg} / \mathrm{dl})^{*}$ & $133.6 \pm 42.5$ & $|3| \pm 46.0$ & 0.8 \\
\hline HDL-C (mg/dl)* & $35.6 \pm 7.7$ & $34.4 \pm 6.2$ & 0.5 \\
\hline Clinical findings at examination* & (Yes/no) & & \\
\hline Arthritis/arthropathy & $6 / 36(16.7 \%)$ & NA & \\
\hline Ocular involvement & $26 / 36(72.2 \%)$ & NA & \\
\hline Neurological involvement & $10 / 36(27.8 \%)$ & NA & \\
\hline Positive pathergy test & $17 / 36(47.2 \%)$ & NA & \\
\hline Vascular involvement & $10 / 36(27.8 \%)$ & NA & \\
\hline Digestive involvement & $3 / 36(8.3 \%)$ & NA & \\
\hline \multicolumn{4}{|l|}{ Medical treatment at inclusion } \\
\hline Colchicine & $36 / 36(100 \%)$ & NA & \\
\hline Prednisolone & $29 / 36(80.6 \%)$ & NA & \\
\hline Azathioprine & I3/17 (76.5\%) & NA & \\
\hline Cyclosporine & $4 / 17$ (23.5\%) & NA & \\
\hline Anticoagulant & $8 / 36(22.2 \%)$ & NA & \\
\hline
\end{tabular}

Notes: "Values are presented as median and interquartile range; *Values are presented as the mean \pm standard deviations. Values in bold indicate statistical significance. Abbreviations: BMI, body mass index; DBP, diastolic blood pressure; SBP, systolic blood pressure; BDCAF, Behçet disease Current Activity Form; CRP, C-reactive protein; HDL-C, high-density lipoprotein; NA, not applicated.

sympathovagal balance ( $\mathrm{LF} / \mathrm{HF}$ ratio) was significantly higher in the BD group as compared to the control group $(\mathrm{p}=0.015)$. However, indices of sympathetic activity (LF component) were comparable between the two groups (Table 2 and Figure 1).
Concerning the morning and night periods, the PNN50, RMSSD and HF component values were significantly reduced in the $\mathrm{BD}$ group compared to the control group. The value of the daytime LF/HF ratio was significantly higher and that of the nighttime LF component was

Table 2 Twenty-Four-Hour Heart Rate Variability Parameters of the Studied Groups

\begin{tabular}{|l|c|c|c|}
\hline HRV Parameters & Behçet's Group (n=36) & Control Group (n=36) & M-W test P value \\
\hline SDNN (ms) & $143.5(117-164.7)$ & $168.5(140.5-189.4)$ & $\mathbf{0 . 0 0 7}$ \\
RMSSD (ms) & $31.5(25.2-42.2)$ & $43(33.3-57)$ & $\mathbf{0 . 0 0 I}$ \\
PNN50 (\%) & $9(5-18.7)$ & $21.5(10.5-32.7)$ & $<0.00 I$ \\
LF $\left(\mathrm{ms}^{2}\right)$ & $802.9(525.7-1076.4)$ & $967.5(694.8-1357.8)$ & 0.08 \\
HF $\left(\mathrm{ms}^{2}\right)$ & $214.7(150.9-440.4)$ & $430.7(262.1-608.4)$ & $\mathbf{0 . 0 0 I}$ \\
LF/HF ratio & $3.4(2.2-5.0)$ & $2.4(1.5-3.4)$ & $\mathbf{0 . 0 1 5}$ \\
\hline
\end{tabular}

Notes: Values are presented as median and interquartile range. Values in bold indicate statistical significance.

Abbreviations: M-W test, Mann-Whitney test; SDNN, standard deviation of all RR intervals; PNN50, percentage of adjacent NN intervals differing by more than 50 ms; RMSSD, root mean-square differences between consecutive RR intervals; LF, low-frequency; HF, high-frequency; LF/HF, low frequency: high frequency ratio. 


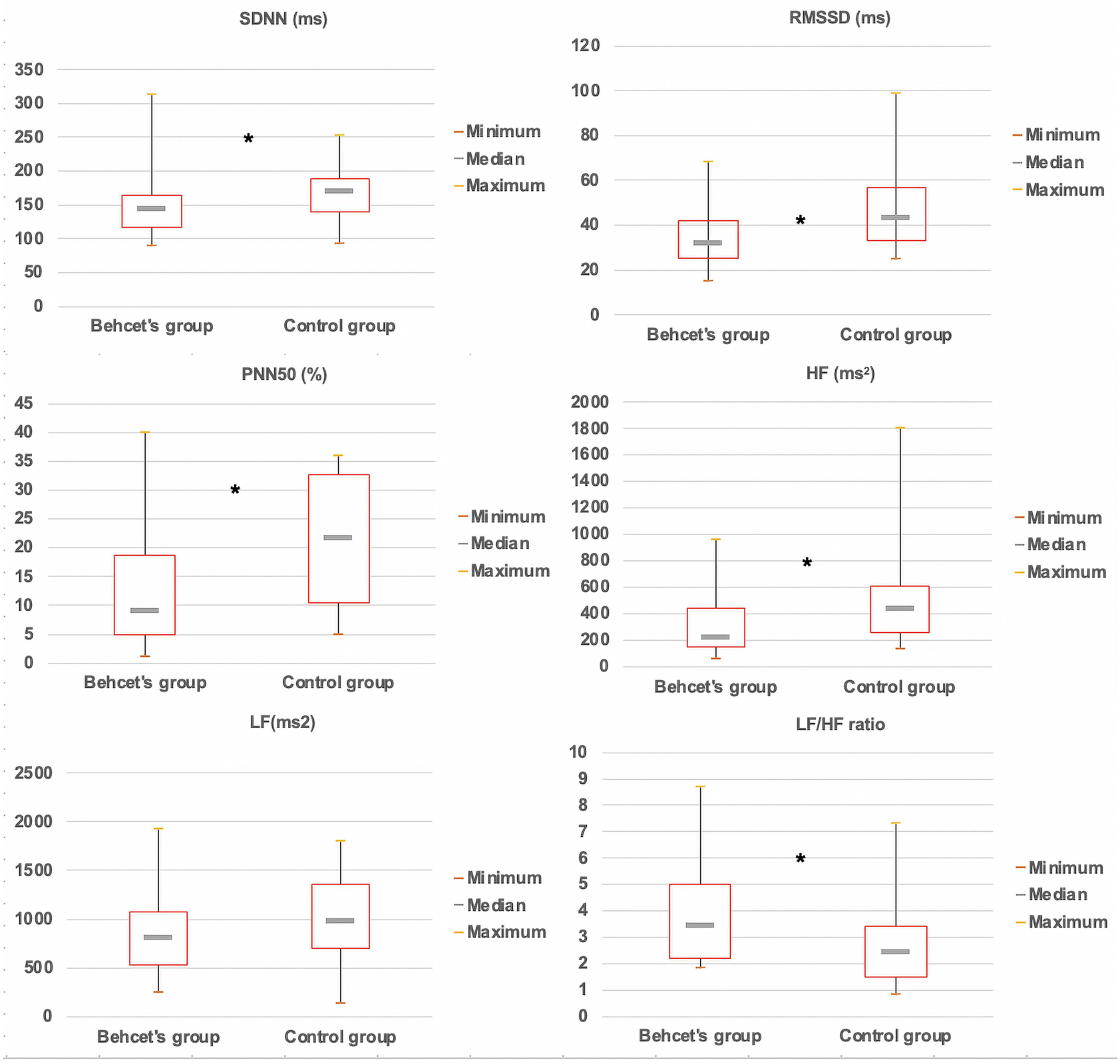

Figure I Boxplots of twenty-four-hour heart rate variability indices of the studies group.

Notes: On each plot, the central mark is the median, the box edges are the 25 th and 75 th percentiles (interquartile range), and the box whiskers extend to the most extreme data points. *Indicates significant differences $(p<0.05)$ between adjacent boxplots.

Abbreviations: SDNN, standard deviation of all normal RR intervals; RMSSD, root mean-square differences between consecutive RR intervals; PNN50, percentage of adjacent NN intervals differing by more than $50 \mathrm{~ms}$; LF/HF, low frequency: high frequency ratio.

significantly lower in the patient group compared with the control group (Table 3).

When comparing diurnal and nocturnal values of different HRV parameters in the patient group, we noted that parameters characterizing sympathetic activity (LF/HF ratio) had significantly higher values in daytime, whereas parameters characterizing parasympathetic activity (RMSSD, P50NN, and HF) had higher values in nighttime (Table 4).
The HRV indices of both patients with active BD and patients that are in remission were calculated and are presented comparatively in Table 5 . There was no significant difference between the two subgroups.

The Spearman correlation between the heart rate variability parameters and CRP, disease activity, disease duration in Behçet's group is presented in Table 6 .

There was no significant correlation of CRP or disease duration with HRV indices in Behçet's subjects. Regarding 
Table 3 Day-Night Variability of the Behcet's Patients and Control Group

\begin{tabular}{|c|c|c|c|}
\hline HRV Parameters & Behçet's Group $(n=36)$ & Control Group $(n=36)$ & $M-W$ test $P$ value \\
\hline \multicolumn{4}{|l|}{ SDNN (ms) } \\
\hline Day & $121.5(10 \mid .5-142)$ & $141.3(105.5-162)$ & 0.083 \\
\hline Night & $104.5(84.2-134)$ & $135(115-146.6)$ & 0.007 \\
\hline \multicolumn{4}{|l|}{ RMSSD (ms) } \\
\hline Day & $29(24.5-39.2)$ & $38(30-52)$ & 0.011 \\
\hline Night & 39 (47-29) & $45.8(38-6 \mid .5)$ & 0.007 \\
\hline \multicolumn{4}{|l|}{ PNN50 (\%) } \\
\hline Day & $7.5(4-15.7)$ & $20(9-29)$ & 0.001 \\
\hline Night & $14.5(6-24.5)$ & $26.5(|6.2-4| .7)$ & 0.001 \\
\hline \multicolumn{4}{|l|}{$\mathrm{LF}\left(\mathrm{ms}^{2}\right)$} \\
\hline Day & $811.6(549.5-1140)$ & $933.5(669.2-1225.6)$ & 0.2 \\
\hline Night & $77 \mid .4$ (468.3-1015.7) & $970.3(794-1485)$ & 0.004 \\
\hline \multicolumn{4}{|l|}{$\mathrm{HF}\left(\mathrm{ms}^{2}\right)$} \\
\hline Day & I8I.7 (I20.4-374.3) & $348.6(218.3-446.7)$ & 0.012 \\
\hline Night & $309.8(198.3-466.6)$ & 483.3 (286-909.6) & 0.011 \\
\hline \multicolumn{4}{|l|}{ LF/HF ratio } \\
\hline Day & $4.1(2.8-6.1)$ & $2.8(1.9-4.2)$ & 0.02 \\
\hline Night & $2.4(1.5-3.2)$ & $2(1.2-3.9)$ & 0.4 \\
\hline
\end{tabular}

Notes: Values are presented as median and interquartile range. Values in bold indicate statistical significance.

Abbreviations: M-W test, Mann-Whitney test; SDNN, standard deviation of all RR intervals; PNN50, percentage of adjacent NN intervals differing by more than 50 ms; RMSSD, root mean-square differences between consecutive RR intervals; LF, low-frequency power; HF, high-frequency power; LF/HF, low frequency: high frequency ratio.

Table 4 Circadian Features of HRV Parameters in Behcet's Disease Patients

\begin{tabular}{|c|c|c|c|}
\hline \multirow[t]{2}{*}{ HRV Parameters } & \multicolumn{2}{|c|}{ Behçet's Group $(n=36)$} & \multirow[t]{2}{*}{$P$ value } \\
\hline & Daytime & Nighttime & \\
\hline SDNN (ms) & $121.5(10 \mid .5-142)$ & $104.5(84.2-134)$ & 0.10 \\
\hline RMSSD (ms) & $29(24.5-39.2)$ & $39(47-29)$ & $<0.001$ \\
\hline PNN50 (\%) & $7.5(4-15.7)$ & $14.5(6-24.5)$ & $<0.001$ \\
\hline $\operatorname{LF}\left(\mathrm{ms}^{2}\right)$ & 811.6 (549.5-1140) & $77 \mid .4(468.3-1015.7)$ & 0.94 \\
\hline $\mathrm{HF}\left(\mathrm{ms}^{2}\right)$ & $181.7(120.4-374.3)$ & $309.8(198.3-466.6)$ & $<0.001$ \\
\hline LF/HF ratio & $4.1(2.8-6.1)$ & $2.4(1.5-3.2)$ & $<0.001$ \\
\hline
\end{tabular}

Notes: Values are presented as median and interquartile range. Values in bold indicate statistical significance.

Abbreviations: SDNN, standard deviation of all normal RR intervals; PNN50, percentage of adjacent NN intervals differing by more than 50 ms; RMSSD, root mean-square differences between consecutive RR intervals; LF, low-frequency; HF, high-frequency; LF/HF, low frequency: high frequency ratio.

Table 5 Comparison of Heart Rate Variability Parameters According to Activity Disease Status

\begin{tabular}{|l|c|c|c|}
\hline Parameters & Behçet's Group Active Disease $(\mathbf{n}=\mathbf{7})$ & Behcet's Group Remission $(\mathbf{n}=29)$ & P value \\
\hline SDNN (ms) & $148(\mid 14-16 I)$ & $142(118-167.5)$ & 0.88 \\
RMSSD (ms) & $40(29-45)$ & $30(25-39.5)$ & 0.24 \\
PNN50 (\%) & $18(7-2 I)$ & $9(5-16.5)$ & 0.27 \\
LF $\left(\mathrm{ms}^{2}\right)$ & $796.5(647-923.4)$ & $809(5|3-1| 4 \mid)$ & 0.82 \\
HF $\left(\mathrm{ms}^{2}\right)$ & $238.2(154.6-663.2)$ & $191.7(131.6-439.4)$ & 0.3 \\
LF/HF ratio & $3.4(1.2-4.1)$ & $3.4(2.3-5.4)$ & 0.18 \\
\hline
\end{tabular}

Note: Values are presented as median and interquartile range.

Abbreviations: SDNN, standard deviation of all normal RR intervals; PNN50, percentage of adjacent NN intervals differing by more than 50 ms; RMSSD, root mean-square differences between consecutive RR intervals; LF, low-frequency power; HF, high-frequency power; LF/HF, low frequency: high frequency ratio. 
Table 6 The Spearman Correlation Between the Heart Rate Variability Parameters and CRP, Disease Activity, Disease Duration in Behçet's Group $(n=36)$

\begin{tabular}{|c|c|c|c|c|c|c|}
\hline & SDNN (ms) & RMSSD (ms) & PNN50 (\%) & $\operatorname{LF}\left(\mathrm{ms}^{2}\right)$ & $\mathrm{HF}\left(\mathrm{ms}^{2}\right)$ & $\operatorname{LF} / \mathrm{HF}\left(\mathrm{ms}^{2}\right)$ \\
\hline CRP & $\begin{array}{l}\text { rho }=-0.143 \\
p=0.4\end{array}$ & $\begin{array}{l}\text { rho }=-0.274 \\
p=0.1\end{array}$ & $\begin{array}{l}\text { rho }=-0.302 \\
P=0.07\end{array}$ & $\begin{array}{l}\text { rho }=-0.184 \\
p=0.28\end{array}$ & $\begin{array}{l}\text { rho }=-0.314 \\
P=0.06\end{array}$ & $\begin{array}{l}\text { rho }=0.305 \\
P=0.07\end{array}$ \\
\hline BDCAF & $\begin{array}{l}\text { rho }=0.029 \\
p=0.8\end{array}$ & $\begin{array}{l}\text { rho }=0.234 \\
p=0.2\end{array}$ & $\begin{array}{l}\text { rho }=0.254 \\
p=0.1\end{array}$ & $\begin{array}{l}\text { rho }=-0.012 \\
p=0.9\end{array}$ & $\begin{array}{l}\text { rho }=0.232 \\
p=0.2\end{array}$ & $\begin{array}{l}\text { rho }=-0.341 \\
\mathbf{p}=\mathbf{0 . 0 4 2}\end{array}$ \\
\hline Disease duration & $\begin{array}{l}\text { rho }=-0.09 \\
p=0.6\end{array}$ & $\begin{array}{l}\text { rho }=-0.060 \\
P=0.7\end{array}$ & $\begin{array}{l}\text { rho }=-0.059 \\
P=0.7\end{array}$ & $\begin{array}{l}\text { rho }=-0.143 \\
P=0.4\end{array}$ & $\begin{array}{l}\text { rho }=-0.166 \\
p=0.3\end{array}$ & $\begin{array}{l}\text { rho }=0.087 \\
P=0.6\end{array}$ \\
\hline
\end{tabular}

Note: Values in bold indicate statistical significance.

Abbreviations: rho, Spearman correlation rho coefficient; SDNN, standard deviation of all normal RR intervals; PNN50, percentage of adjacent NN intervals differing by more than 50 ms; RMSSD, root mean-square differences between consecutive RR intervals; LF, low-frequency power; HF, high-frequency power; LF/HF, ratio between LF and HF; CRP, C-reactive protein; BDCAF, Behçet disease Current Activity Form.

the activity score, there was a significant negative moderate correlation between $\mathrm{LF} / \mathrm{HF}$ ratio and BDCAF score $($ rho $=-0.341, \mathrm{p}=0.042)$.

\section{Discussion}

In this study, patients with Behcet's disease had reduced parasympathetic activity compared with controls. However, the physiological circadian fluctuation of autonomic function was preserved.

Previous studies have used a variety of approaches to demonstrate autonomic involvement in Behcet's disease, including analysis of cardiac recovery index, sympathetic skin response (SSR) and R-R interval variation (RRIV), heart rate variability and heart rate turbulence (HRT) studies. ${ }^{8,12-14}$ In spite of several studies, whether the autonomic nervous system is impaired in this disease and which components of the ANS are involved, is still a matter of debate.

Heart rate variability is a measure of cardiac autonomic modulation (CAM) which provides a quantitative evaluation of sympathetic and parasympathetic influences on the heart. ${ }^{15}$ There are scant previous studies that have evaluated HRV in this disease up to date.

In the present study, when comparing HRV parameters between the patient group and control subjects, we found a significant reduction in parameters reflecting parasympathetic tone in the time (PNN50, RMSSD) and frequency (HF) domains in the Behcet's disease group. Parameters exploring the sympathetic system (LF) were comparable between the two groups. These results suggest the presence of subclinical parasympathetic ANS involvement in patients with Behçet's disease.
Our results were consistent with those of Ozdemir et al ${ }^{16}$ who evaluated ANS function in a population of 45 patients with BD using 24-hour time-domain analysis of HRV and found a decrease in RMSSD and PNN50 values in patients with $\mathrm{BD}$ as compared to controls, indicating a dysfunction of parasympathetic innervation. They also noted that the decrease in parasympathetic tone was positively correlated with disease duration (PNN50: $\mathrm{r}=0.58, \mathrm{p}<0.01$; RMSSD: $r=0.58, p<0.01$ ), a result that was not confirmed in our study. The mean duration of the disease was $36.9 \pm 23$ months in this study while it was $104.75 \pm 101.83$ months in our study, and this difference may be responsible for the discrepancy in the results.

In the same context, Aksu et $\mathrm{al}^{17}$ studied ANS function in a group of $54 \mathrm{BD}$ patients and showed that all timedomain HRV parameters were impaired in the Behcet's disease group compared with the control group, particularly those reflecting parasympathetic activity (RMSSD, PNN50 and SDNN indices). In this study, all patients included had less than 10 years of diagnosis and the majority of patients were female.

Aksoyek et $\mathrm{al}^{18}$ performed a short-term (5-minute) frequency analysis of HRV in 71 patients with BD and 26 matched healthy controls. HRV parameters were measured in the supine and standing positions. Lower HF values and higher $\mathrm{LF}$ and $\mathrm{LF} / \mathrm{HF}$ ratio values were observed in patients with $\mathrm{BD}$ as compared to controls. Authors concluded that sympathetic control of the heart rate in $\mathrm{BD}$ patients was increased, whereas parasympathetic modulation was decreased.

In contrast, Kirimli et a ${ }^{19}$ found a significant decrease in SDNN and PNN50 in patients with Behcet's disease compared with controls. However, the frequency domain 
parameters were comparable between the two groups. These results could not suggest a clear autonomic abnormality. In agreement with this study, Erol et $\mathrm{al}^{20}$ found no significant difference in time and frequency domain parameters between patients and controls, implying normal autonomic function.

Another relevant study by $\mathrm{Akci}$ et $\mathrm{al}^{21}$ including 40 patients with Behcet's disease and 20 age- and sexmatched healthy subjects assessed cardiac autonomic function by heart rate turbulence. Cardiac autonomic function was not found to be impaired in patients with Behcet disease compared with healthy controls. These results may be explained by the fact that the HRT indices may indicate aspects of the autonomic nervous system that are different from those reflected by the HRV parameters.

In healthy subjects, HRV exhibits a circadian rhythm, with an increase in heart rate and $\mathrm{LF} / \mathrm{HF}$ ratio during the day and an increase in HF component at night. ${ }^{22}$

The day-night physiological changes of HRV parameters reflect the sympathetic predominance during the day and the vagal predominance during the night. Our study showed that the circadian pattern of heart rate variability was preserved in Behcet's disease patients.

CRP and BDCAF are parameters of disease activity. In this study, we did not find a relationship between BDCAF score and HRV parameters, except for LF/HF ratio. This finding could be explained by the fact that this score probably does not provide a complete measure of disease activity. Similarly, changes in ANS parameters were not associated with levels of inflammatory markers (CRP) in our study.

Pathogenesis of autonomic dysfunction in Behcet disease is still unknown; it might be caused by chronic inflammation of the nerves which results in suppression of nerve signal transmission, ${ }^{13}$ immune function with direct damage of baroreceptors and nerves mediated by cytokines and vasculitis of the vasa-nervorum system or compressing nerve fibers by fibrosis. ${ }^{18}$

By reviewing the various studies carried out in this context, we spotted several factors that can explain the difference in the results. Firstly, the duration of disease was different among studies. Secondly, the levels of inflammation are unknown in some studies ${ }^{17-21}$ and the proportion of patients in active phase was not reported in all studies. Some, like us, included patients in the active phase or in remission, whereas in others only recruited patients in remission. ${ }^{16-18,21}$ Another point to be raised is the effect of drug on ANS. In our study, all patients with BD were on low-dose colchicine on a regular basis and approximately half of patients were taking immunosuppressive drugs, whereas in the study of Ozdemir et al ${ }^{16}$ all treatments were stopped 3 weeks before the evaluation of HRV. Colchicine had been known to have chronotropic cardiac effects in vitro. However, we do not yet know the autonomic functional changes that occur in actual patients taking colchicine. ${ }^{23}$ On the other hand, the effect of this immunosuppressive therapy on HRV parameters is also unknown. We believe that taking this into consideration. As last, genetic, and constitutional differences could have an impact and explain the observed discrepancies.

On the basis of our findings, we can only confirm the presence of subclinical parasympathetic ANS involvement in Behcet's disease patients. Published literature data are discordant regarding LF component and LF/HF ratio. In some studies, LF was a quantitative marker of sympathetic modulations, and so $\mathrm{LF} / \mathrm{HF}$ ratio was thus a reflection of sympathovagal balance. Whereas in other studies, the LF component of HRV has been found to be not sensitive enough to assess the sympathetic activity. ${ }^{24}$ Indeed, heart rate oscillations in the LF zone are related to the activity of the baroreflex system ${ }^{25}$ and both the parasympathetic and sympathetic systems are thought to be involved. As a consequence, the $\mathrm{LF} / \mathrm{HF}$ ratio should be interpreted with caution, taking into consideration all these limitations and contributing factors.

Further studies including other parameters of HRV, such as Baevsky's stress index, which is a valid parameter strongly correlated to sympathetic activity, may be helpful for better assessment of sympathetic part of ANS. ${ }^{25}$

Another point to raise was the lack of study of the processes of regulation of peripheral vascular tone. The study of the interaction between the regulation of the heart and peripheral blood flow expands the possibilities of assessing autonomic dysfunction. This could be studied by Photoplethysmography (PPG) which is a non-invasive functional vascular investigation technique reflecting beatto-beat relative blood volume changes in the microvascular bed of peripheral tissues and a useful tool for the study of autonomic control of the peripheral vascular tone. ${ }^{26}$

As known, there is strong evidence for the role of the autonomic system in the pathogenesis of ventricular arrhythmias, ${ }^{27}$ autonomic dysfunction can increase the risk of ventricular arrhythmias in Behçet disease. ${ }^{4,28,29}$

As the early recognition of abnormalities in the ANS may be very important to prevent excessive morbidity, regular monitoring by Holter recording seems important to detect and manage possible rhythmic abnormalities at an early stage. 
The main limitations of our study are on one hand the small size of the study population, and on the other hand, the fact that it is a cross-sectional study, limiting our ability to assess causality; thus, longitudinal studies and repeated measurements are necessary to draw conclusive findings.

\section{Conclusion}

In conclusion, compared with the controls, patients with BD displayed decreased HRV in both the time and frequency domains, reflecting decreased parasympathetic tone. Our results provide evidence for impaired cardiac autonomic functions in BD patients despite the absence of overt cardiac involvement and symptoms. Further studies are needed to elucidate the prognostic significance and clinical implications of impaired autonomic functions in patients with Behcet's disease.

\section{Highlights Section}

- Patients with Behcet disease, despite no cardiovascular involvement, have reduced parasympathetic activity compared with controls. Then they have an increased risk of ventricular arrhythmia.

- Circadian rhythm of heart rate variability is preserved in Behcet disease patients.

- Heart rate variability, as practical tool, can be an interesting approach for the rhythmic follow-up of Behcet disease patients.

\section{Disclosure}

The authors report no conflicts of interest for this work.

\section{References}

1. Sakane T, Takeno M, Suzuki N, Inaba G. Behçet's disease. $N$ Engl $J$ Med. 1999;341(17):1284-1291. PMID: 10528040. doi:10.1056/ NEJM199910213411707

2. Alpsoy E. Behçet's disease: a comprehensive review with a focus on epidemiology, etiology and clinical features, and management of mucocutaneous lesions. J Dermatol. 2016;43(6):620-632. PMID: 27075942. doi:10.1111/1346-8138.13381

3. Davatchi F, Shahram F, Chams-Davatchi C, et al. Behcet's disease: from East to West. Clin Rheumatol. 2010;29(8):823-833. PMID 20354748. doi:10.1007/s10067-010-1430-6

4. Göldeli O, Ural D, Komsuoğlu B, Ağaçdiken A, Dursun E, Cetinarslan B. Abnormal QT dispersion in Behçet's disease. Int $J$ Cardiol. 1997;61(1):55-59. PMID: 9292333. doi:10.1016/ s0167-5273(97)00120-4

5. Schiff S, Moffatt R, Mandel WJ, Rubin SA. Acute myocardial infarction and recurrent ventricular arrhythmias in Behcet's syndrome. Am Heart J. 1982;103(3):438-440. PMID: 7064781. doi:10.1016/00028703(82)90289-7

6. Shen MJ, Zipes DP. Role of the autonomic nervous system in modulating cardiac arrhythmias. Circ Res. 2014;114(6):1004-1021. PMID: 24625726. doi:10.1161/CIRCRESAHA.113.302549
7. Rajendra Acharya U, Paul Joseph K, Kannathal N, Lim CM, Suri JS. Heart rate variability: a review. Med Biol Eng Comput. 2006;44 (12):1031-1051. PMID: 17111118. doi:10.1007/s11517-006-0119-0

8. Behbahani S, Shahram F. Electrocardiogram and heart rate variability assessment in patients with common autoimmune diseases: a methodological review. Turk Kardiyol Dern Ars. 2020;48 (3):312-327. PMID: 32281951. doi:10.5543/tkda.2019.21112

9. Criteria for diagnosis of Behçet's disease. International Study Group for Behçet's disease. Lancet. 1990;335(8697):1078-1080. PMID: 1970380.

10. Hamuryudan V, Fresko I, Direskeneli H, et al. Evaluation of the Turkish translation of a disease activity form for Behçet's syndrome. Rheumatology. 1999;38(8):734-736. PMID: 10501421. doi:10.1093/ rheumatology/38.8.734

11. Task Force of the European Society of Cardiology and the North American Society of Pacing and Electrophysiology. Heart rate variability: standards of measurement, physiological interpretation and clinical use. Circulation. 1996;93(5):1043-1065. PMID: 8598068. doi:10.1161/01.CIR.93.5.1043

12. Kaya EB, Yorgun H, Akdogan A, et al. Heart-rate recovery index is impaired in Behçet's disease. Tex Heart Inst J. 2009;36(4):282-286. PMID: 19693299; PMCID: PMC2720298.

13. Borman P, Tuncay F, Kocaoğlu S, Okumuş M, Güngör E, Ekşioğlu M. The subclinic autonomic dysfunction in patients with Behçet disease: an electrophysiological study. Clin Rheumatol. 2012;31(1):41-47. PMID: 21597928. doi:10.1007/s10067-011-17639

14. Karataş GK, Onder M, Meray J. Autonomic nervous system involvement in Behçet's disease. Rheumatol Int. 2002;22(4):155-159. PMID: 12172955. doi:10.1007/s00296-002-0220-0

15. Stein PK, Bosner MS, Kleiger RE, Conger BM. Heart rate variability: a measure of cardiac autonomic tone. Am Heart J. 1994;127 (5):1376-1381. PMID: 8172068. doi:10.1016/0002-8703(94)90059-0

16. Ozdemir R, Sezgin AT, Topal E, Kutlu R, Barutcu I, Gullu H. Findings of ambulatory blood pressure monitoring and heart rate variability in patients with Behcet's disease. Am J Cardiol. 2003;92 (5):646-648. doi:10.1016/s0002-9149(03)00747-1

17. Aksu T, Guler ET, Kaya V, et al. Comparison of electrocardiographic findings and holter recordings in patients with Behcet's disease and healthy volunteers. Int J Cardiovasc Res. 2015;4:1. doi:10.4172/ 2324-8602.1000196

18. Aksoyek S, Aytemir K, Ozer N, Ozcebe O, Oto A. Assessment of autonomic nervous system function in patients with Behcet's disease by spectral analysis of heart rate variability. J Auton Nerv Syst. 1999;77(2-3):190-194. PMID: 11130963. doi:10.1016/S01651838(99)00045-4

19. Kirimli O, Aslan O, Göldeli O, et al. Heart rate variability, late potentials and QT dispersion as markers of myocardial involvement in patients with Behçet's disease. Can J Cardiol. 2000;16 (3):345-351. PMID: 10744798.

20. Erol T, Tekin A, Tufan M, et al. Autonomic neural control of the cardiovascular system in patients with Behçet's disease in the absence of neurological involvement. Clin Rheumatol. 2012;31 (10):1499-1504. doi:10.1007/s10067-012-2045-x

21. Akci O, Aldemir M, Yaman F, et al. Assessment of cardiac autonomic function by using heart rate turbulence in Behcet's disease. World J Cardiovasc Surg. 2014;4:193-199. doi:10.4236/ wjcs.2014.411028

22. Bonnemeier H, Wiegand UKH, Brandes A, et al. Circadian profile of cardiac autonomic nervous modulation in healthy subjects. J Cardiovasc Electrophysiol. 2003;14(8):791-799. doi:10.1046/ j.1540-8167.2003.03078.x

23. Klein I. Colchicine stimulates the rate of contraction of heart cells in culture. Cardiovasc Res. 1983;17(8):459-465. PMID: 6616518. doi:10.1093/cvr/17.8.459 
24. Ali MK, Liu L, Chen JH, Huizinga JD. Optimizing autonomic function analysis via heart rate variability associated with motor activity of the human colon. Front Physiol. 2021;12:619722. PMID: 34267670; PMCID: PMC8275990. doi:10.3389/fphys.2021.619722

25. Robbe HW, Mulder LJ, Rüddel H, Langewitz WA, Veldman JB, Mulder G. Assessment of baroreceptor reflex sensitivity by means of spectral analysis. Hypertension. 1987;10(5):538-543. PMID: 3666866. doi:10.1161/01.hyp.10.5.538

26. Karavaev AS, Borovik AS, Borovkova EI, et al. Low-frequency component of photoplethysmogram reflects the autonomic control of blood pressure. Biophys J. 2021;120(13):2657-2664. PMID: 34087217. doi:10.1016/j.bpj.2021.05.020
27. Tsuji H, Larson MG, Venditti FJ Jr, et al. Impact of reduced heart rate variability on risk for cardiac events. The Framingham Heart Study. Circulation. 1996;94(11):2850-2855. PMID: 8941112. doi:10.1161/ 01.cir.94.11.2850

28. Kahn JK, Sisson JC, Vinik AI. QT interval prolongation and sudden cardiac death in diabetic autonomic neuropathy. J Clin Endocrinol Metab. 1987;64 (4):751-754. PMID: 3818902. doi:10.1210/jcem-64-4-751

29. Aytemir K, Ozer N, Aksoyek S, Ozcebe O, Kabakci G, Oto A. Increased QT dispersion in the absence of QT prolongation in patients with Behcet's disease and ventricular arrhythmias. Int J Cardiol. 1998;67(2):171-175. PMID: 9891952. doi:10.1016/ s0167-5273(98)00322-2

\section{Publish your work in this journal}

The International Journal of General Medicine is an international, peer-reviewed open-access journal that focuses on general and internal medicine, pathogenesis, epidemiology, diagnosis, monitoring and treatment protocols. The journal is characterized by the rapid reporting of reviews, original research and clinical studies across all disease areas. The manuscript management system is completely online and includes a very quick and fair peer-review system, which is all easy to use. Visit http://www.dovepress.com/ testimonials.php to read real quotes from published authors. 